\title{
Upregulation of Rho7 in the temporal lobe tissue of humans with intractable epilepsy
}

\author{
XINGLONG YANG ${ }^{1,2}$, BAIYUAN YANG ${ }^{3}$, HAONAN LI $^{4}$ and HUI REN ${ }^{1}$ \\ ${ }^{1}$ Department of Geriatric Neurology, First Affiliated Hospital of Kunming Medical University, \\ Kunming, Yunnan 650032; ${ }^{2}$ Department of Neurology, West China Hospital, Sichuan University; \\ ${ }^{3}$ Department of Neurology, Chengdu Seventh People's Hospital, Chengdu, Sichuan 610041; \\ ${ }^{4}$ Department of Neurology, The People's Hospital of Chuxiong State, Chuxiong, Yunan 675000, P.R. China
}

Received August 17, 2016; Accepted May 5, 2017

DOI: $10.3892 / \mathrm{mmr} .2017 .7787$

\begin{abstract}
Patients with intractable epilepsy (IE) exhibit an increased risk of premature death, psychosocial dysfunction and decreasing quality of life. The present study aimed to investigate the alteration in the expression of Rho7 in brain tissue from patients with IE, and to examine the association between Rho7 protein expression and IE. Temporal lobe samples were collected from the temporal lobes of 33 patients with IE patients and 10 age- and gender-matched histologically healthy controls. Immunohistochemical staining was conducted to assess the number of Rho7-positive cells. In addition, double-label immunofluorescent staining was performed to examine the cellular localization of Rho7. The protein expression of Rho7 was examined using western blotting. Marked immunoreactivity for Rho7 was detected in the IE group, while faint and scattered immunoreactive staining was observed in the control group. The count of Rho7 positive cells in the IE patients was significantly increased compared with the control subjects $(23.47 \pm 3.9 \%$ vs. $12.09 \pm 1.05 \%$; P<0.01). Double-label immunofluorescent staining indicated that Rho7 was primarily expressed in the cell membrane and cytoplasm, and colocalized with neuron-specific enolase. Western blot analysis demonstrated that the expression of Rho7 in the IE group was significantly increased compared with the control group $(0.41 \pm 0.031$ vs. $0.25 \pm 0.025 ; \mathrm{P}<0.01)$. The results of the present study demonstrated that upregulation of Rho7 immunoreactivity occurs in the brains of patients with IE, suggesting that Rho7 may be associated with the progression of IE or act as a potential treatment target.
\end{abstract}

Correspondence to: Professor Hui Ren, Department of Geriatric Neurology, First Affiliated Hospital of Kunming Medical University, 295 Xichang Road, Kunming, Yunnan 650032, P.R. China E-mail: hrhuiren@163.com

Key words: Rho7, intractable epilepsy, temporal lobe, neuron

\section{Introduction}

Epilepsy has developed into a serious worldwide health concern, accounting for $\sim 1 \%$ of the global disease burden (1). Epilepsy is a chronic condition, or a group of chronic conditions, in which abnormal neuronal discharge leads to transient disturbance of cerebral function and recurrent seizures. It has been reported that one-third of all epilepsy patients exhibit medically intractable epilepsy (IE), which accounts for $\sim 80 \%$ of the overall cost of epilepsy $(2,3)$. Patients with IE exhibit an increased risk of premature death, psychosocial dysfunction and poor quality of life (4). A previous study concluded that a number of etiological, pharmacological, demographic and genetic factors may contribute to the development of IE (5). Temporal lobe epilepsy (TLE) is the most common type of focal epilepsy, and $\sim 35 \%$ of patients with TLE experience chronic seizures that are not responsive to antiepileptic-drugs (AEDs) (5,6). Therefore, the identification of potential drug targets for IE is urgently required in order to develop improved clinical solutions.

At present, an increasing number of studies have been conducted to investigate the molecular mechanisms of IE, and the identification of abnormally-expressed molecules may aid the development of novel targets for the treatment of patients with IE. It has been reported that the expression of the microRNAs miR-199a-5p and miR-199b-5p was markedly decreased, whereas the levels of HIF-1 $\alpha$ were upregulated, in epileptic brain tissues compared with those from control subjects (7). A deficiency of glutathione peroxidase was previously identified in patients with IE(8). Mitochondrially-encoded cytochrome $\mathrm{b}$, ubiquinol-cytochrome $\mathrm{c}$ reductase binding protein, ubiquinol-cytochrome c reductase core protein I and ubiquinol-cytochrome $\mathrm{c}$ reductase hinge protein were identified to be potential drug targets for IE through analysis of protein-protein interactions and drug-target relationships (9). However, the mechanisms underlying the pathogenesis of IE remain unclear.

Rho family small GTPases serve important roles in the regulation of the cytoskeleton, phagocytosis, membrane trafficking, cell migration and subsequent morphological changes in neurons (10-13). Rho family GTPases have been reported to be associated with neuronal network formation (14). Rho7 
(additionally termed Rnd2 and RhoN), a Rho family small GTPase, is specifically expressed in brain neurons (15). Rho7 has been observed to act as a regulator of certain processes in neurons that are associated with IE $(16,17)$. Considering the roles of Rho7 in neurons, it may be hypothesized that Rho7 may be associated with IE. However, few studies have investigated the alterations in the expression of Rho7 in patients with IE.

In the present study, the expression level and location of Rho7 protein was assessed in temporal lobe tissues obtained from patients with IE and healthy controls, using immunohistochemistry, double-label immunofluorescence staining and western blotting.

\section{Materials and methods}

Brain sample collection. The present study was performed in accordance with the Declaration of Helsinki (18). Brain tissue samples were collected from the temporal lobes of 33 patients with IE who had received neurosurgery between January 2003 and December 2008 at the Newbridge Hospital of the Third Military Medical University (Chongqing, China), 999 Brain Hospital of Guangdong (Guangzhou, China) and The First Affiliated Hospital of Chongqing University of Medical Science (Chongqing, China). All patients exhibited clinical TLE, which was independently evaluated by two neuropathologists. The resected temporal lobe tissues were obtained from the suspected area of seizure onset. Seizure types were classified according to the 1981 International Classification of Epileptic Seizures of the International League against Epilepsy (19). The inclusion criteria for the patients were as follows: i) All patients exhibited the typical electroencephalogram and clinical manifestation of epilepsy; ii) AED therapy had failed in all patients with maximum tolerable doses of at least three AEDs (including phenytoin, valproic acid, carbamazepine, phenobarbital, topiramate, oxcarbazepine, clonazepam, gabapentin and lamotrigine); iii) the patients exhibited no apparent underlying cause of epilepsy; iv) physical inspection of the nervous system indicated no focal physical signs, and pathology findings in the resected tissues demonstrated no direct focus responsible for the epilepsy; v) chemotherapy or magnetic resonance imaging exhibited no abnormalities, except for hippocampal sclerosis; vi) no patients had contraindications of surgery according to the preoperative evaluation; and vii) written consent was obtained from a family member.

For comparison, 10 healthy temporal lobe tissue samples were used as controls, eight of which were histologically normal temporal tissues from autopsy specimens with craniocerebral trauma, and the other two specimens were from two persons who had succumbed to an accident. All of the samples matched the conditions listed below: i) The subjects suffered no epilepsy or any other neurology disease; ii) temporal tissue structure was integral as confirmed by pathological analysis; and iii) written informed consent was obtained from a family member.

All the experiments in the present study were approved by the ethics committee of the Newbridge Hospital of the Third Military Medical University.

Immunohistochemistry. The temporal tissue was extracted from each participant, and then fixed with $4 \%$ paraformaldehyde at $4^{\circ} \mathrm{C}$ overnight. Next, the tissue was dehydrated with gradient ethanol, vitrified with xylene and embedded in paraffin wax. The slices were cut into slices of 3-5- $\mu \mathrm{m}$ thickness using a microtome. The sections were prepared for the further studies.

The Rho7 expression in patients with IE and controls was evaluated using an immunohistochemistry assay. All paraffin-embedded sections were deparaffinized in xylene and rehydrated with graded ethanol. Antigens were retrieved using $0.01 \mathrm{M}$ citric acid ( $\mathrm{pH} \mathrm{6.0)}$ for $15 \mathrm{~min}$ at $92-97^{\circ} \mathrm{C}$. Following treatment with $3 \% \mathrm{H}_{2} \mathrm{O}_{2}$ for 15 min to quench endogenous peroxidase activity, the sections were blocked in 5\% normal rabbit serum (cat. no. ZLI-9025; OriGene Technologies, Inc., Beijing, China) at room temperature for $30 \mathrm{~min}$ to reduce non-specific binding, followed by incubation with the primary antibody (rabbit anti-human Rho7; cat. no. sc-28565; 1:50; Santa Cruz Biotechnology, Inc., Dallas, TX, USA) at $4^{\circ} \mathrm{C}$ overnight. Following washing three times with PBS, the sections were incubated with biotinylated secondary goat anti-rabbit antibody (1:500; cat. no. ZB-2010; Zhongshan Golden Bridge Biotechnology Co., Ltd.) at room temperature for $30 \mathrm{~min}$ and reacted with avidin-biotin peroxidase complex solution (cat. no. PK-4001; OriGene Technologies, Inc.) at $30^{\circ} \mathrm{C}$ for 30 min. 3,3'-diaminobenzidine (Sigma-Aldrich; Merck KGaA, Darmstadt, Germany) was used as the final chromogen, and hematoxylin was utilized as the nuclear counterstain at room temperature for $5 \mathrm{~min}$. For negative controls, the primary or secondary antibody was omitted and replaced with PBS or non-immune immunoglobulin (Ig)G to evaluate the experimental procedure and reagent quality. The Olympus PM20 automatic microscope (Olympus Corporation, Tokyo, Japan) and the TCFY-2050 pathology system (Yuancheng, Inc., Beijing, China) were utilized for image acquisition. Images for each sample were automatically analyzed and semi-quantitated using the Motic Med 6.0 CMIAS pathology image analysis system (Motic China Group Co., Ltd., Xiamen, China). A total of 10 visual fields were randomly selected for each section under light microscopy (magnification, x400). A total of 100 cells in each field were selected and the number of positive cells was calculated. The positive cell proportion was calculated as follows: Positive cell proportion=(number of positive cells/100)x100. All the experiments were performed three times.

Double-label immunofluorescence. Double-label immunofluorescence was performed to detect the cellular colocalization of Rho7 and neuron-specific enolase (NSE), an enolase isoform expressed in the central nervous system, which has been widely used as a neuronal marker (20). Following dewaxing, rehydration and antigen retrieval (as described above), tissue sections (3-5 $\mu \mathrm{m}$ ) were incubated in calf serum (OriGene Technologies, Inc.) at room temperature for $1 \mathrm{~h}$, and subsequently in normal goat serum (OriGene Technologies, Inc.) at room temperature for $5 \mathrm{~h}$. The sections were incubated with rabbit anti-human Rho7 antibody (1:100; cat. no. sc-28565; Santa Cruz Biotechnology, Inc.) or mouse monoclonal anti-human NSE antibody (1:200; cat. no.ZM-0203; OriGene Technologies, Inc.) at $4^{\circ} \mathrm{C}$ overnight, followed by incubation with goat anti-rabbit tetramethylrhodamine isothiocyanate-conjugated secondary antibody (1:100; cat. no. ZF-0311; OriGene Technologies, Inc.) or goat anti-mouse fluorescein isothiocyanate-conjugated 
secondary antibody (1:100; cat. no. ZF-0312; OriGene Technologies, Inc.) in the dark at room temperature for $2 \mathrm{~h}$. The sections were subsequently cover-slipped, sealed and dried overnight. Fluorescent-stained sections were examined using confocal laser scanning microscopy (magnification, x400; Leica Microsystems GmbH, Wetzlar, Germany). Images were captured and processed using the Olympus Micro Image analysis software system (version 4.0; Olympus Corporation).

Western blotting. The samples were randomly selected from the temporal lobe tissues of patients with IE $(n=4)$ and controls $(n=2)$ to cut into small pieces. The samples were homogenized in buffer including protease inhibitors $(15 \mu \mathrm{g} / \mathrm{ml}$ aprotinin and $1 \mathrm{mM}$ phenylmethylsulfonyl fluoride), and centrifuged at $16,000 \mathrm{x} \mathrm{g}$ and $4^{\circ} \mathrm{C}$ for $5 \mathrm{~min}$. The crude nuclear pellet was lysed in a lysis buffer [20 mM Tris, (pH 7.4), 1\% Triton X-100, 10\% glycerol, $137 \mathrm{mM} \mathrm{NaCl}, 2 \mathrm{mM}$ EDTA, $25 \mathrm{mM} \beta$-glycerophosphate, $1 \mathrm{mM}$ sodium orthovanadate, $2 \mathrm{mM}$ pyrophosphate, $1 \mathrm{mM}$ phenylmethylsulfonyl fluoride, $10 \mu \mathrm{g} / \mathrm{ml}$ leupeptin] for $30 \mathrm{~min}$ and centrifuged at $16,000 \mathrm{x} \mathrm{g}$ and $4^{\circ} \mathrm{C}$ for $15 \mathrm{~min}$. The protein concentration was determined using a Coomassie Blue G-250 kit (Sigma-Aldrich; Merck KGaA). The samples (40 $\mu \mathrm{g})$ were resolved using SDS-PAGE on a $10 \%$ gel and electrotransferred onto a polyvinylidene fluoride membrane (DuPont, Wilmington, DE, USA). Subsequently, the membranes were blocked with $3 \%$ bovine serum albumin (Sigma-Aldrich; Merck KGaA) for $2 \mathrm{~h}$ at room temperature. The membranes were incubated with rabbit anti-human Rho7 (1:100; cat. no. sc-28565; Santa Cruz Biotechnology, Inc.) or mouse anti-human $\beta$-actin (1:1,000; cat. no. PR-0255; OriGene Technologies, Inc.) antibodies at $37^{\circ} \mathrm{C}$ for $1 \mathrm{~h}$. Following washing with PBS, the membranes were incubated with goat anti-rabbit horseradish peroxidase (HRP)-IgG (1:5,000; cat. no. RABHRP1; Sigma-Aldrich; Merck KGaA) or goat anti-mouse HPR-IgG (1:5,000; cat. no. RABHRP2; Sigma-Aldrich; Merck KGaA) secondary antibodies at $37^{\circ} \mathrm{C}$ for $1 \mathrm{~h}$. The immunoreactive bands were visualized using an enhanced chemiluminescence (ECL) system (DuPont). Relative quantification of the ECL signal on X-ray film was analyzed using Labworks ${ }^{\mathrm{TM}}$ Analysis Software (version 4.5; UVP, Inc., Upland, CA, USA). Following global background subtraction, gray densities of Rho7 bands were normalized to the $\beta$-actin band for each lane. All the experiments were performed in triplicate.

Data analysis. Quantitative data are expressed as the mean \pm standard deviation, and qualitative data are expressed as a percentage of subjects. All statistical analyses were performed using SPSS (version 19.0; IBM Corp., Armonk, NY, USA). Student's t-test was used to compare the quantitative data and $\chi^{2}$ test was used to compare the qualitative data between the IE and control groups. $\mathrm{P}<0.05$ was considered to indicate a statistically significant difference.

\section{Results}

Age and gender composition of the IE group and control group. The treatment history of the included 33 patients is summarized in Table I. The age and sex distributions were compared between the IE and control groups, and the results are presented in Table II. The mean age was $24.4 \pm 8.68$ and
$26.4 \pm 6.75$ years in the IE and control groups, respectively. There were more male patients $(51.6 \%)$ in the IE group, while there were more women (60\%) in the control group. However, there were no significant differences in age (t-test; $\mathrm{P}=0.606)$ or sex $\left(\chi^{2}\right.$ test; $\left.P=0.523\right)$ between the patients with IE and the control subjects.

Rho7 is overexpressed in temporal tissue of IE patients, as demonstrated by immunohistochemistry. Temporal tissue sections from IE and control subjects were immunohistochemically stained. The results of the present study demonstrated that marked immunoreactivity for Rho7 was detected in the IE group, while faint and scattered immunoreactive staining was observed in the control group (Fig. 1A). The positive cell count of Rho7 in the IE patients was significantly increased compared with the control subjects $(23.47 \pm 3.9 \%$ vs. $12.09 \pm 1.05 \%$; $\mathrm{P}<0.01$; Fig. 1B).

Rho7 is colocalized with NSE in neurons of the temporal lobe. Double-label immunofluorescence staining was performed to examine the cellular localization of Rho7 in the temporal lobe tissues of patients with IE and control subjects. The double-label immunofluorescence staining demonstrated that Rho7-positive cells (red) co-localized with NSE (green) in neurons in the temporal lobe tissues (Fig. 2). According to the results, Rho7 was primarily expressed in the neuronal cytoplasm and colocalized with NSE in the temporal lobe tissues. Compared with control group, the Rho7-positive cells were notably increased in the IE group, exhibiting stronger green fluorescence.

Rho7 is overexpressed in the temporal tissue of patients with $I E$, as demonstrated by western blotting. In order to further confirm the altered expression of Rho7 in IE temporal tissues, the expression of Rho7 in the temporal lobe tissues obtained from patients with IE and controls was examined using western blotting. Rho7 (26 kDa) and $\beta$-actin (42 kDa internal control) proteins were detected in the present study. The results indicated that the expression of Rho7 protein in the IE group was increased compared with the control group (representative results are presented in Fig. 3A). In addition, the differences in relative protein expression were significant $(0.41 \pm 0.031$ vs. $0.25 \pm 0.025$; $\mathrm{P}<0.01$; Fig. 3B).

\section{Discussion}

Patients with medically-confirmed IE are at an increased risk of depression and suicide, particularly patients with more severe and frequent seizures (21). It has been reported that the prevalence of depression and suicidality in patients with medically intractable seizures may be 50 and $19 \%$, respectively $(22,23)$. Therefore, it is necessary to investigate the mechanisms and abnormally expressed molecules in IE, which may improve the understanding of IE and provide novel research targets. Rho proteins are involved in neuronal and non-neuronal cell migration, axonal outgrowth and guidance, and also dendrite formation (24). Rho7 is specifically expressed in brain neurons, and is able to induce synapse growth and sprouting (25-29). In the present study, it was demonstrated that Rho7 protein was expressed in the temporal lobe tissue of patients with IE and 
Table I. Treatment history of the 33 patients in the epilepsy group.

\begin{tabular}{|c|c|c|c|c|c|c|c|}
\hline $\mathrm{N}$ & $\mathrm{A}$ & $\mathrm{G}$ & $\mathrm{D}$ & Antiepiletic drugs & Seizure type & Imaging & Pathology \\
\hline 1 & 32 & M & 12 & CBZ, PHT, PB, TCM & SPS, CPS & fcd & $\mathrm{g}, \mathrm{nl}$ \\
\hline 2 & 38 & $\mathrm{M}$ & 16 & CBZ, PHT, PB, TCM & SPS , CPS & rhs & ac \\
\hline 3 & 25 & M & 8 & CBZ, VPA, PHT, TPM & SPS, CPS & $\mathrm{n}$ & $\mathrm{g}, \mathrm{nd}$ \\
\hline 4 & 12 & $\mathrm{~F}$ & 8 & VPA, CBZ, TCM & CPS & fcd & $\mathrm{g}, \mathrm{nd}, \mathrm{nl}$ \\
\hline 5 & 12 & $\mathrm{~F}$ & 10 & $\mathrm{CBZ}, \mathrm{PB}, \mathrm{VPA}$ & SPS, CPS & $\mathrm{n}$ & $\mathrm{nl}, \mathrm{g}$ \\
\hline 6 & 17 & $\mathrm{M}$ & 10 & CBZ, PHT, VPA, TPM & SPS & $\mathrm{n}$ & $\mathrm{g}$ \\
\hline 7 & 25 & $\mathrm{~F}$ & 4 & $\mathrm{CBZ}, \mathrm{PB}, \mathrm{VPA}$ & SPS, CPS & $\mathrm{n}$ & $\mathrm{g}$ \\
\hline 8 & 23 & $\mathrm{~F}$ & 15 & CBZ, PHT, VPA, PB & GTCS & $\mathrm{n}$ & $\mathrm{g}, \mathrm{nl}$ \\
\hline 9 & 28 & M & 5 & CBZ, VPA, PHT, TPM & GTCS & $\mathrm{n}$ & $\mathrm{nl}$ \\
\hline 10 & 32 & $\mathrm{~F}$ & 19 & VPA, CBZ, PB, TPM & CPS & $\mathrm{n}$ & $\mathrm{g}, \mathrm{nd}$ \\
\hline 11 & 30 & $\mathrm{M}$ & 8 & VPA, CBZ, TPM & SGS & fcd & ac \\
\hline 12 & 44 & $\mathrm{~F}$ & 20 & PHT, VPA, LMT & CPS & rhs & $\mathrm{g}$ \\
\hline 13 & 17 & M & 10 & CBZ, PHT, PB, VPA & SGS & $\mathrm{n}$ & $\mathrm{g}, \mathrm{nl}$ \\
\hline 14 & 56 & $\mathrm{~F}$ & 10 & PHT, CBZ, GBA & SGS & fcd & $\mathrm{nl}, \mathrm{nd}$ \\
\hline 15 & 22 & M & 10 & CBZ, VPA, LMT & SGS & rhs & $\mathrm{g}, \mathrm{na}$ \\
\hline 16 & 28 & M & 6 & TPM, CBZ, VPA & CPS & rhs & $\mathrm{g}, \mathrm{nl}, \mathrm{nd}$ \\
\hline 17 & 27 & $\mathrm{~F}$ & 20 & PHT, PB, VPA, LMT & CPS & lhs & $\mathrm{g}$ \\
\hline 18 & 33 & $\mathrm{~F}$ & 10 & VPA, TPM, CBZ & SGS & $\mathrm{n}$ & $\mathrm{nl}, \mathrm{g}$ \\
\hline 19 & 14 & $\mathrm{~F}$ & 8 & VPA, PB, LMT & SGS & $\mathrm{n}$ & $\mathrm{ac}$ \\
\hline 20 & 26 & $\mathrm{~F}$ & 12 & CBZ, PHT, VPA & SGS & $\mathrm{n}$ & $\mathrm{nl}, \mathrm{g}$ \\
\hline 21 & 27 & $\mathrm{~F}$ & 14 & PHT, VPA, LMT & SGS & $\mathrm{n}$ & $\mathrm{g}$ \\
\hline 22 & 47 & M & 29 & PB, PHT, VPA, TPM, LMT6 & SGS & $\mathrm{n}$ & ac \\
\hline 23 & 28 & M & 3 & VPA, PB, CBZ & SGS & $\mathrm{n}$ & $\mathrm{nl}, \mathrm{nd}, \mathrm{g}$ \\
\hline 24 & 29 & $\mathrm{~F}$ & 27 & VPA, CBZ, LMT & SGS & $\mathrm{n}$ & $\mathrm{g}, \mathrm{nl}$ \\
\hline 25 & 22 & $\mathrm{~F}$ & 10 & CBZ, PHT, Pb & SPS, CPS & $\mathrm{n}$ & $\mathrm{g}, \mathrm{nl}$ \\
\hline 26 & 24 & M & 5 & CBZ, PHT, TPM, ТСМ & SPS, GTCS & $\mathrm{n}$ & $\mathrm{g}$ \\
\hline 27 & 31 & M & 10 & CBZ, PB, TPM, TCM & SPS, CPS & $\mathrm{n}$ & $\mathrm{g}$ \\
\hline 28 & 20 & M & 12 & CBZ, PHT, TPM, ТCM & SPS , CPS & $\mathrm{n}$ & $\mathrm{nl}, \mathrm{g}$ \\
\hline 29 & 15 & $\mathrm{~F}$ & 6 & CBZ, PB, TPM, TCM & SPS, CPS & $\mathrm{n}$ & ac \\
\hline 30 & 19 & M & 10 & CBZ, PB, TPM & SPS, CPS & $\mathrm{n}$ & $\mathrm{ac}$ \\
\hline 31 & 37 & M & 11 & CBZ, VPA, PB & SPS , CPS & $\mathrm{n}$ & $\mathrm{nl}$, nd \\
\hline 32 & 28 & M & 10 & $\mathrm{CBZ}, \mathrm{PB}, \mathrm{TPM}$ & SPS, CPS & $\mathrm{n}$ & $\mathrm{g}, \mathrm{nd}$ \\
\hline 33 & 46 & $\mathrm{~F}$ & 11 & CBZ, PHT, TPM, TCM & SPS, CPS & $\mathrm{n}$ & $\mathrm{nl}$ \\
\hline
\end{tabular}

N, number; A, age; D, duration of epilepsy; G, gender; M, male; F, female; CBZ, carbamazepine; PB, phenobarbital; PHT, phenytoin; VPA, valproate; TPM, topiramate; TCM, traditional Chinese medicine; CPS, complex partial seizure; SPS, simple partial seizures; GTCS, generalized tonic-clonic seizure; fcd, focal cortical malacia; n, normal; nl, neuronal loss; nd, neuronal degeneration; g, gliosis; ac, astrocytosis; rhs, right hippocampus sclerosis; lhs, left hippocampus sclerosis.

Table II. Age and sex composition of the IE and control groups.

\begin{tabular}{lccc}
\hline & IE $(\%), n=33$ & Control $(\%), n=10$ & P-value \\
\hline Age & $24.4 \pm 8.68$ & $26.4 \pm 6.75$ & 0.606 \\
Sex & & & \\
Female & $16(48.4)$ & $6(60)$ & 0.523 \\
Male & $17(51.6)$ & $4(40)$ & \\
\hline
\end{tabular}

Data are presented as the mean \pm standard deviation. IE, intractable epilepsy. control subjects. However, the expression of Rho7 in the IE group was significantly increased compared with the control group.

According to the results of the immunohistochemical analysis and immunofluorescence staining, Rho7 was expressed in the neuronal membrane and cytoplasm, and co-localized with the neuronal marker NSE. Neurons migrate extensively to reach their permanent location in the nervous system (30). The correct positioning of neurons during development is the basis for proper brain function (31). It has also been reported that abnormal neuronal migration may lead to abnormal cortical 

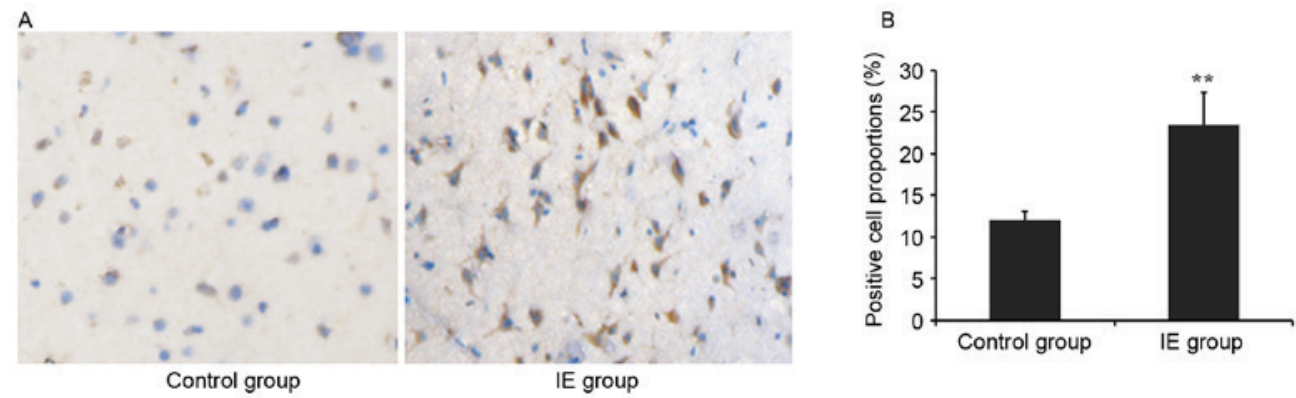

Figure 1. Rho7 is overexpressed in temporal tissue of patients with IE, as demonstrated by immunohistochemistry. (A) Immunohistochemistry of Rho7-positive expression in the temporal tissue of patients with IE and control subjects (magnification, x400). (B) Positive cell proportions of Rho7 in IE and control groups. Data are presented as the mean + standard deviation. ${ }^{* *} \mathrm{P}<0.01$ vs. control group. IE, intractable epilepsy.
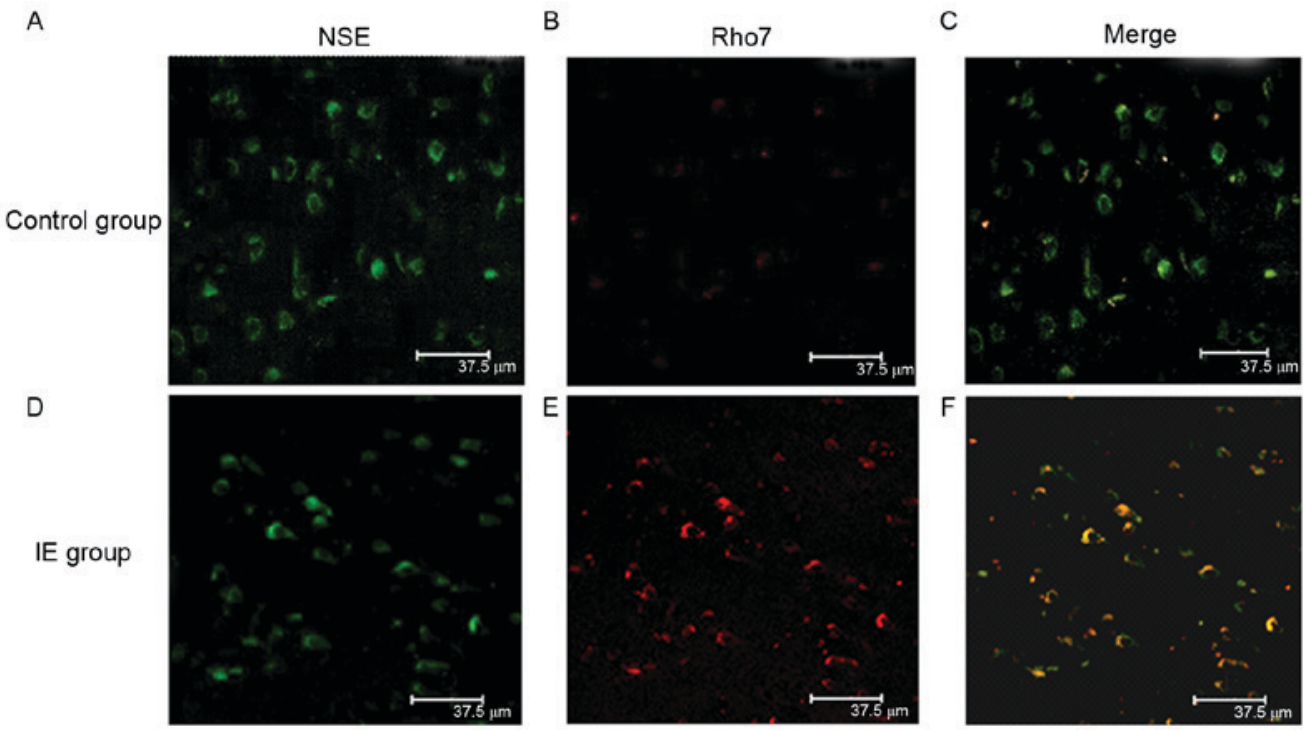

Figure 2. Rho7 is colocalized with NSE in the neurons of the temporal lobe tissues. (A) FITC staining for NSE in the control group. (B) TRITC staining for Rho7 in the control group. (C) Overlay of Rho7 and NSE expression in the control group. (D) FITC staining for NSE in the IE group. (E) TRITC staining for Rho7 in the IE group. (F) Overlay of Rho7 and NSE expression in the IE group. NSE, neuron-specific enolase; FITC, fluorescein isothiocyanate; TRITC, tetramethylrhodamine isothiocyanate; IE, intractable epilepsy.

A

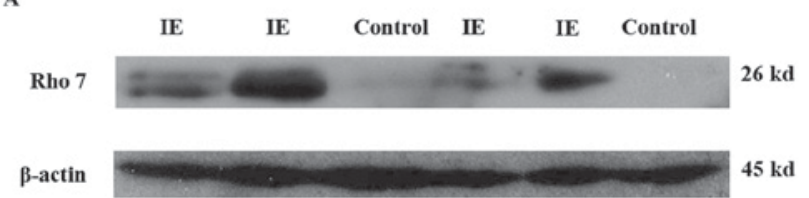

B

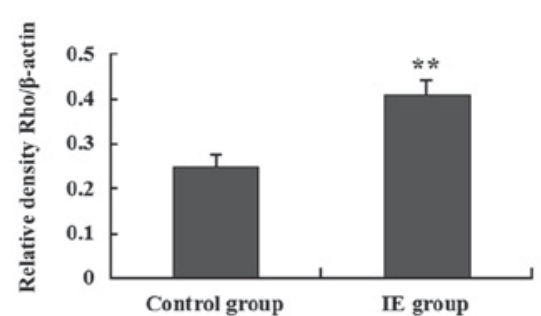

Figure 3. Rho7 expression is increased in the temporal lobe of patients with IE compared with control subjects. (A) Western blot analysis of Rho7 expression in patients with IE and control subjects. (B) Relative protein expression was calculated to demonstrate the fold change of Rho7, normalized to $\beta$-actin. A total of two samples were used from the control group and four samples from the IE group. Data are presented as the mean + standard deviation, $n=3$. ${ }^{* *} \mathrm{P}<0.01$ vs. control group. IE, intractable epilepsy. function, including epilepsy $(32,33)$. Rho7 is able to regulate the migration and morphological alterations associated with the development of pyramidal neurons in vivo (16). In addition, excessive hypertrophic pyramidal neurons have been reported in patients with IE who underwent brain resections (34). Heng et al (17) identified that Rho7 acted as an essential regulator of neuronal migration, disorders of which are reported to be associated with IE (35). Therefore, the overexpression of Rho7 may be associated with IE. Ras homolog family member A (RhoA) activity is associated with neuronal migration, and certain molecules are able to regulate neuronal migration via RhoA inhibition (36). Rho7 inhibits RhoA signaling in cortical neurons and regulates migration by inhibiting RhoA activity in a variety of cellular compartments (37). Therefore, it was hypothesized that Rho7 may regulate neuronal migration in IE by acting as a RhoA inhibitor. However, the specific mechanism of Rho7 in IE was not investigated in the present study, and future studies are required to elucidate this.

In addition, the axon of the neuron is associated with epilepsy. The axon initial segment (AIS) serves a role in neuronal excitability, as the site of action potential initiation is 
located here (38). It has been hypothesized that AIS plasticity may alter the excitability of a neuron in response to the environment, enhancing or attenuating neuronal sensitivity $(39,40)$. A previous study demonstrated that Rho7 is involved in the molding of neurons by translocating from the cytoplasm to the cell membrane (41). At the onset of epilepsy, Rho7 may activate RhoA and promote the detachment of the axon from a target cell (42), and subsequently induce neurite branching via rapostlin, an effector of Rho7 $(43,44)$. Pragmin, another effector of Rho7, is activated to promote the growth of neurite branches, which will form abnormal synaptic connections (45). These abnormal connections account for the abnormal neural network that is the pathological basis of epilepsy (46). In the present study, it was observed that the expression of Rho7 in the IE group was significantly increased compared with the control group. Based on pathological investigations, it was hypothesized that Rho7 may be involved in the formation of abnormal neuronal networks and be associated with the pathogenesis of IE. Notably, Rho7 was also observed to be expressed in astrocytes and deposited in the synapse (data not shown). As Rho GTPases are associated with the regulation of the cytoskeleton in a variety of cells, including astrocytes $(47,48)$, it was hypothesized that Rho7 may be associated with the growth and development of astrocytes. However, the role of Rho7 in the astrocytes of patients with IE requires further investigation.

In conclusion, the results of the present study demonstrated that Rho7 expression was increased in the neurons of patients with IE compared with control samples, which indicated that Rho7 may be associated with the progression of IE or act as a potential target for IE treatment. However, the localization and expression levels of Rho7 were only detected in temporal lobe tissues. Further studies are required to investigate the association between Rho7 and IE, and to investigate the underlying mechanism through which this protein is involved in IE.

\section{References}

1. Engel J Jr, McDermott MP, Wiebe S, Langfitt JT, Stern JM, Dewar S, Sperling MR, Gardiner I, Erba G, Fried I, et al: Early surgical therapy for drug-resistant temporal lobe epilepsy: A randomized trial. JAMA 307: 922-930, 2012.

2. Berg AT: Understanding the delay before epilepsy surgery: Who develops intractable focal epilepsy and when? CNS Spectr 9: 136-144, 2004.

3. Begley CE, Famulari M, Annegers JF, Lairson DR, Reynolds TF, Coan S, Dubinsky S, Newmark ME, Leibson C, So EL and Rocca WA: The cost of epilepsy in the United States: An estimate from population-based clinical and survey data. Epilepsia 41: 342-351, 2000

4. Hao XT, Wong IS and Kwan P: Interrater reliability of the international consensus definition of drug-resistant epilepsy: A pilot study. Epilepsy Behav 22: 388-390, 2011.

5. Wassenaar M, Leijten FS, Egberts TC, Moons KG and Uijl SG: Prognostic factors for medically intractable epilepsy: A systematic review. Epilepsy Res 106: 301-310, 2013.

6. Engel J Jr; International League Against Epilepsy (ILAE): A proposed diagnostic scheme for people with epileptic seizures and with epilepsy: Report of the ILAE task force on classification and terminology. Epilepsia 42: 796-803, 2001.

7. Jiang G, Zhou R, He X, Shi Z, Huang M, Yu J and Wang X: Expression levels of microRNA-199 and hypoxia-inducible factor-1 alpha in brain tissue of patients with intractable epilepsy. Int J Neurosci 126: 326-334, 2016.

8. Nazıroğlu M and Yürekli VA: Effects of antiepileptic drugs on antioxidant and oxidant molecular pathways: Focus on trace elements. Cell Mol Neurobiol 33: 589-599, 2013.
9. Chu H, Zhou X, Liu G, Lv M, Wang Y, Liu L, Li X, Sun P, Zhu Y, Sun C, et al: Network-based detection of disease modules and potential drug targets in intractable epilepsy. Journal: 132-140, 2014.

10. Kawauchi T: Regulation of cell adhesion and migration in cortical neurons: Not only Rho but also Rab family small GTPases. Neuron 2: 36-40, 2011.

11. Etienne-Manneville S and Hall A: Rho GTPases in cell biology. Nature 420: 629-635, 2002.

12. Moon SY and Zheng Y: Rho GTPase-activating proteins in cell regulation. Trends Cell Biol 13: 13-22, 2003.

13. Ridley AJ: Rho GTPases and cell migration. J Cell Sci 114: 2713-2722, 2001

14. Negishi $\mathrm{M}$ and Katoh $\mathrm{H}$ : Rho family GTPases as key regulators for neuronal network formation. J Biochem 132: 157-166, 2002.

15. Decourt B, Bouleau Y, Dulon D and Hafidi A: Expression analysis of neuroleukin, calmodulin, cortactin, and Rho7/Rnd2 in the intact and injured mouse brain. Brain Res Dev Brain Res 159: 36-54, 2005.

16. Nakamura K, Yamashita Y, Tamamaki N, Katoh H, Kaneko T and Negishi M: In vivo function of Rnd2 in the development of neocortical pyramidal neurons. Neurosci Res 54: 149-153, 2006.

17. Heng JI, Nguyen L, Castro DS, Zimmer C, Wildner H, Armant O, Skowronska-Krawczyk D, Bedogni F, Matter JM, Hevner R and Guillemot F: Neurogenin 2 controls cortical neuron migration through regulation of Rnd2. Nature 455: 114-118, 2008.

18. World Medical Association: World Medical Association Declaration of Helsinki. Ethical principles for medical research involving human subjects. Bull World Health Organ 79: 373-374, 2001.

19. Dreifuss FE, Bancand J, Henriksen O, Rubio-Donnadieu F, Seino M and Penry JK: Commission on classification and terminology of the international league against epilepsy, proposal for revised clinical and electroencephalographic classification of epileptic seizures. Epilepsia: 489-501, 1981.

20. Fang M, Liu GW, Pan YM, Shen L, Li CS, Xi ZQ, Xiao F, Wang L, Chen D and Wang XF: Abnormal expression and spatiotemporal change of Slit2 in neurons and astrocytes in temporal lobe epileptic foci: A study of epileptic patients and experimental animals. Brain Res 1324: 14-23, 2010.

21. Kimiskidis VK, Triantafyllou NI, Kararizou E, Gatzonis SS, Fountoulakis KN, Siatouni A, Loucaidis P, Pseftogianni D, Vlaikidis N and Kaprinis GS: Depression and anxiety in epilepsy: The association with demographic and seizure-related variables. Ann Gen Psychiatry 6: 28, 2007.

22. Kanner AM, Soto A and Gross-Kanner H: Prevalence and clinical characteristics of postictal psychiatric symptoms in partial epilepsy. Neurology 62: 708-713, 2004.

23. Boylan LS, Flint LA, Labovitz DL, Jackson SC, Starner K and Devinsky O: Depression but not seizure frequency predicts quality of life in treatment-resistant epilepsy. Neurology 62: 258-261, 2004.

24. Govek EE, Hatten ME and Van Aelst L: The role of Rho GTPase proteins in CNS neuronal migration. Dev Neurobiol 71: 528-553, 2011.

25. McLoughlin HS, Fineberg SK, Ghosh LL, Tecedor L and Davidson BL: Dicer is required for proliferation, viability, migration and differentiation in corticoneurogenesis. Neuroscience 223: 285-295, 2012.

26. Fujita H, Katoh $\mathrm{H}$, Ishikawa $\mathrm{Y}$, Mori $\mathrm{K}$ and Negishi M: Rapostlin is a novel effector of Rnd2 GTPase inducing neurite branching. J Biol Chem 277: 45428-45434, 2002.

27. Kakimoto T, Katoh $\mathrm{H}$ and Negishi M: Identification of splicing variants of Rapostlin, a novel RND2 effector that interacts with neural Wiskott-Aldrich syndrome protein and induces neurite branching. J Biol Chem 279: 14104-14110, 2004.

28. Tsuchiya D, Kitamura Y, Takata K, Sugisaki T, Taniguchi T, Uemura K, Miki H, Takenawa T and Shimohama S: Developmental expression of neural Wiskott-Aldrich syndrome protein (N-WASP) and WASP family verprolin-homologous protein (WAVE)-related proteins in postnatal rat cerebral cortex and hippocampus. Neurosci Res 56: 459-469, 2006.

29. Li J and Anton E: Rnd-ing up RhoA activity to link neurogenesis with steps in neuronal migration. Dev Cell 20: 409-410, 2011

30. Marin O and Rubenstein JL: Cell migration in the forebrain. Annu Rev Neurosci 26: 441-483, 2003.

31. Ayala R, Shu T and Tsai LH: Trekking across the brain: The journey of neuronal migration. Cell 128: 29-43, 2007.

32. Guerrini R and Parrini E: Neuronal migration disorders. Neurobiol Dis 38: 154-166, 2010 
33. Chai X, Münzner G, Zhao S, Tinnes S, Kowalski J, Häussler U, Young C, Haas CA and Frotscher M: Epilepsy-induced motility of differentiated neurons. Cerebral Cortex 24: 2130-2140, 2014.

34. Wang DD, Blümcke I, Coras R, Zhou WJ, Lu DH, Gui QP, Hu JX, Zuo HC,Chen SY and Piao YS: Sturge-weber syndrome is associated with cortical dysplasia ILAE Type IIIc and excessive hypertrophic pyramidal neurons in brain resections for intractable epilepsy. Brain Pathol 25: 248-255, 2014.

35. Roper SN, Gilmore RL and Houser CR: Experimentally induced disorders of neuronal migration produce an increased propensity for electrographic seizures in rats. Epilepsy Res 21: 205-219, 1995

36. Tang J, Ip JP, Ye T, Ng YP, Yung WH, Wu Z, Fang W, Fu AK and Ip NY: Cdk5-dependent Mst3 phosphorylation and activity regulate neuronal migration through RhoA inhibition. J Neurosci 34 7425-7436, 2014

37. Pacary E, Heng J, Azzarelli R, Riou P, Castro D, Lebel-Potter M, Parras C, Bell DM, Ridley AJ, Parsons M and Guillemot F: Proneural transcription factors regulate different steps of cortical neuron migration through Rnd-mediated inhibition of RhoA signaling. Neuron 69: 1069-1084, 2011.

38. Wimmer VC, Reid CA, So EY, Berkovic SF and Petrou S: Axon initial segment dysfunction in epilepsy. J Physiol 588: 1829-1840, 2010.

39. Yoshimura T and Rasband MN: Axon initial segments: Diverse and dynamic neuronal compartments. Curr Opin Neurobiol 27 96-102, 2014

40. Kuba H, Oichi Y and Ohmori H: Presynaptic activity regulates $\mathrm{Na}(+)$ channel distribution at the axon initial segment. Nature 465 1075-1078, 2010.
41. Katoh H, Harada A, Mori K and Negishi M: Socius is a novel Rnd GTPase-interacting protein involved in disassembly of actin stress fibers. Mol Cell Biol 22: 2952-2964, 2002.

42. Wennerberg K, Forget MA, Ellerbroek SM, Arthur WT, Burridge K, Settleman J, Der CJ and Hansen SH: Rnd proteins function as RhoA antagonists by activating p190 RhoGAP. Current Biol 13: 1106-1115, 2003.

43. Goh LL and Manser E: The GTPase-deficient Rnd proteins are stabilized by their effectors. J Biol Chem 287: 31311-31320, 2012.

44. Wakita Y, Kakimoto T, Katoh H and Negishi M: The F-BAR protein Rapostlin regulates dendritic spine formation in hippocampal neurons. J Biol Chem 286: 32672-32683, 2011

45. Tanaka H, Katoh $\mathrm{H}$ and Negishi M: Pragmin, a novel effector of Rnd2 GTPase, stimulates RhoA activity. J Biol Chem 281: 10355-10364, 2006.

46. Vaessen MJ, Braakman HM, Heerink JS, Jansen JF, Debeij-van Hall MH, Hofman PA, Aldenkamp AP and Backes WH Abnormal modular organization of functional networks in cognitively impaired children with frontal lobe epilepsy. Cerebral Cortex 23: 1997-2006, 2013.

47. Guasch RM, Blanco AM, Pérez-Aragó A, Miñambres R, Talens-Visconti R, Peris B and Guerri C: RhoE participates in the stimulation of the inflammatory response induced by ethanol in astrocytes. Exp Cell Res 313: 3779-3788, 2007.

48. Höltje M, Hoffmann A, Hofmann F, Mucke C, Grosse G, Van Rooijen N, Kettenmann H, Just I and Ahnert-Hilger G: Role of Rho GTPase in astrocyte morphology and migratory response during in vitro wound healing. J Neurochem 95: 1237-1248, 2005 . 